\title{
Problem Drug-related Behavior and Discontinuation of Opioids Following the Introduction of an Opioid Management Program
}

\author{
Lucinda A. Grande, MD, Ellen Campbell Thompson, BA, Margaret A. Au, MS, \\ Devin Sawyer, MD, Laura-Mae Baldwin, MD, MPH, and Roger Rosenblatt, ${ }^{\dagger} M D, M P H$
}

Purpose: Problem drug-related behavior (PDB) among patients on chronic opioid therapy may reflect an opioid use disorder. This study assessed PDB prevalence and the relationship between PDB and ongoing prescription of opioids at a primary care clinic that implemented a multifaceted opioid management program.

Methods: A chart review of patients in a chronic opioid registry assessed prevalence of different types of PDB over 2 years, and whether opioids were prescribed during the last 3 months of the 2 -year study period among patients with different levels of PDB.

Results: Among 233 registry patients, 84.1\% exhibited PDB; $45.5 \%$ exhibited $\geq 3$ types of PDB. At the end of 2 years, most registry patients were still prescribed opioids, though patients with $\geq 3$ types of PDB were less likely than those without PDB to be prescribed opioids $(62.3 \%$ vs. $78.4 \%, P=0.016)$.

Conclusions: PDB was pervasive in this population of patients on chronic opioid therapy. Those with the most PDB, and thus with the greatest likelihood of opioid use disorder and its social and medical consequences, were the least likely to be prescribed opioids by the clinic after 2 years. Given the rising rates of illicit opioid use in the U.S., it is important that clinics work closely with their patients who display PDB, systematically assess them for opioid use disorder, and offer evidence-based treatment. (J Am Board Fam Med 2016;29:718-726.)

Keywords: Ambulatory Care Facilities; Analgesics, Opioid, Opioid-Related Disorders; Prevalence; Primary Health Care; Registries; Safety-Net Providers; Substance Abuse Detection

From 1990 to 2010, a steady increase in the use of prescription opioid medications for the treatment of chronic pain in the United States was accompanied by a parallel increase in the rate of deaths resulting from prescription opioid overdose which reached a level rivaling that of deaths from motor vehicle accidents. ${ }^{1}$ Professional organizations, ${ }^{2-4}$ legislative bodies, ${ }^{5}$ and the Centers for Disease

This article was externally peer reviewed.

Submitted 19 February 2016; revised 10 August 2016; accepted 15 August 2016.

From the Department of Family Medicine, University of Washington School of Medicine, Seattle (LAG, ECT, MAA, L-MB, RR); and Providence St. Peter Family Medicine, Olympia, WA (DS).

†Died December 12, 2014.

Funding: This study was supported by the Washington State Life Science Discovery Fund (WSU-LSDF subcontract 109212-G002562, Principal Investigator, John Roll) and a University of Washington Medical Student Research Training Grant (ECT).
Control and Prevention ${ }^{6}$ have sought to reduce harm from opioid medications by publishing opioid management guidelines and setting limits related to the prescribing of opioids.

While death from overdose is the most prominent potential harm of chronic opioid therapy, less catastrophic concerns that nevertheless cause distress for patients are the uncomfortable withdrawal symptoms and increased sensitivity to pain often associated with this category of medication. ${ }^{7}$ This distress can lead patients to crave and use more medication than prescribed, and some engage in

Prior presentation: Data from this study were presented as a poster at the Association for Medical Education and Research in Substance Abuse 38th annual conference, November 6-8, 2014, San Francisco, CA.

Conflict of interest: none declared.

Corresponding author: Lucinda A. Grande, MD, Pioneer Family Practice, 5130 Corporate Ctr Ct SE, Lacey, WA 98503 (E-mail: cgrande@uw.edu). 
maladaptive behaviors to obtain more of the drug. Such problem drug-related behaviors (PDBs) can include repeatedly requesting early medication refills, engaging in deception such as untruthfully reporting medications as lost or stolen, or displaying uncontrolled anger when denied a prescription. $^{8}$

Continued prescribing of opioids to patients exhibiting PDB may have undesirable consequences. For providers and clinic staff repeatedly encountering $\mathrm{PDB}$, consequences may include inefficiency, stress, and compassion fatigue. ${ }^{9}$ Ultimately, the supply of primary care physicians may be adversely affected: negative experiences during medical training with patients exhibiting PDB while receiving chronic opioid therapy may discourage students ${ }^{10}$ and residents ${ }^{9}$ from a career in primary care. For patients-many of whom may be struggling with an opioid use disorder-continuation of opioid prescriptions may reinforce their maladaptive behaviors. Failure of providers to identify and treat an opioid use disorder in these patients risks the patients' psychosocial deterioration, transition to illicit opioid use, medical complications, and death from overdose. ${ }^{11}$

Estimates of the prevalence of PDBs among patients on chronic opioid therapy have varied widely. ${ }^{12-14}$ Most previous studies have been performed in pain specialty settings and have attempted to identify only the most severe problems. There is little information about the range of clinically significant problems encountered in the primary care setting, where most patients on chronic opioid therapy receive their prescriptions.

This retrospective cohort study describes the scope and types of PDB in a primary care clinic that initiated a prescription opioid management program, and the relationship between the level of PDB and opioid prescribing after 2 years of the program.

\section{Methods \\ Setting}

This study took place in a primary care safety net clinic within a family medicine residency, with 30 mostly part-time providers (27 physicians and 3 nurse practitioners) serving 8,000 patients in an urban region with a population of roughly 140,000 .

\section{Prescription Opioid Management Program}

In December 2009 the clinic's management identified problematic behaviors of patients prescribed chronic opioid therapy as an area of concern. A quality improvement project was initiated to improve the care of these patients. Clinic management appointed a project workgroup that included 2 physicians (LAG and DS), a nurse practitioner, 2 registered nurses, 3 certified medical assistants, and a staff assistant. The workgroup developed plans for a program to increase adherence to opioid management guidelines. ${ }^{2-4}$ The program included a registry of patients prescribed opioids and a set of opioid-related support services offered by clinic staff to providers and registry patients. The program did not include provider education or rules constraining the prescribing of opioids; individual prescribing decisions were left to the providers.

Beginning in December 2009 the workgroup developed a registry of patients who were routinely prescribed opioid medications for chronic noncancer pain. Information technology staff used the electronic medical record to select patients with $\geq 3$ opioid prescriptions over the previous year. From that list, primary care providers identified patients whom they considered to be prescribed opioids for chronic noncancer pain. The staff assistant added the International Classification of Diseases, 9th Revision (ICD-9), diagnosis code 338.4 (chronic pain syndrome) to the active problem list of each patient in this subset, thereby designating registry status. The clinic staff and providers added more patients to the registry over time as they were identified, but they did not remove the diagnosis code from an existing patient's problem list, even if opioids were no longer prescribed.

The clinic's support services included phone calls by clinic staff to patients to schedule visits for opioid monitoring via (1) semiannual provider visits, (2) random quantitative urine drug testing (UDT) twice yearly, and (3) annual treatment agreement updates. Primary care providers retained the privilege of excusing their patients from this schedule. Additional support services were optional periodic telephone check-ins by medical assistants to monitor pain and function (at an interval mutually agreeable to the patient, provider, and medical assistant), and a protocol-driven prescription refill program for patients when approved by their provider. The workgroup chose to apply the monitoring schedule without risk stratification of 
patients because of their perception of a high risk for substance use disorders among this patient population. All urine samples were sent to a commercial laboratory that performed quantitative analysis by mass spectroscopy after initial screening by immunoassay (Ameritox, Midland, TX).

After the opioid management program was initiated, the project workgroup anecdotally observed a reduction in the prescribing of opioids to patients with frequent problematic behaviors. To determine whether this observation was valid, members of the workgroup collaborated with a larger academic team in 2012 to design a study that would describe the PDB of the registry patients and opioid prescribing patterns. The hypothesis was that after 2 years of the program, discontinuation of opioid prescribing was more common among patients with more PDB.

\section{Data Development}

The study cohort included individuals who were (1) designated as part of the registry (having a diagnosis of ICD-9, code 338.4 on the active problem list), (2) prescribed opioids by the start of the study period (at least 1 prescription on or before December 1, 2009), and (3) chronically prescribed opioids during the study period (prescriptions for at least 6 individual months during the 2-year study period). Patients in hospice or with a painful cancer, based on review of medical record notes and problem list diagnoses, were excluded.

Beginning in 2013, the primary researcher (LAG) used a standard template to extract deidentified data for all cohort patients from the electronic medical record for the study period (December 1 , 2009 through November 30, 2011). The extraction process included review and interpretation of each patient's clinic visit notes, telephone encounters, prescription records, and patient documents (e.g., reports from the emergency department and consultants). Extracted data included (1) demographic information (age at the beginning of the study period, sex, race [white vs nonwhite]), (2) employment status at the end of the study period, (3) insurance status at the end of the study period (publicly insured [Medicare or Medicaid] vs other or noninsured), (4) evidence of smoking, depression, anxiety, or post-traumatic stress disorder at any time during the study period, (5) information about opioid medications prescribed by providers in the clinic (drug name, date, dose, quantity, and number of refills), (6) UDT results, (7) treatment agreement dates, (8) clinic visit dates, and (9) the existence of different types of PDB. A patient's baseline duration of prescribed opioid therapy was calculated as the number of months between the patient's first opioid prescription in the electronic medical record (which was implemented in November 2005) and the start of the study period (December 1, 2009).

Opioid formulations containing codeine, fentanyl, hydrocodone, hydromorphone, methadone, morphine, oxycodone, and oxymorphone were included. Tramadol and propoxyphene were excluded. By convention, prescriptions in this clinic during the study period were written for 30 days, with or without refills. The total morphine-equivalent dose (MED) was calculated for all prescriptions for each patient for each month using a standard conversion chart. ${ }^{4}$ All prescription refills were assumed to have been dispensed unless chart notes indicated otherwise, and refills were attributed to successive months following the month of the prescription. The patient's average MED was calculated as the sum of the MED in each nonzero month over the study's 2 years, divided by the total number of nonzero months.

PDB types were defined to reflect the range of problematic behaviors reported in other studies of patients on chronic opioid therapy, ${ }^{12-14}$ since no gold standard exists for determining problems with prescription opioids. A PDB type was designated if at least 1 occurrence was identified, except for the "early refill" behavior, which required more than 1 prescription refill at least 5 days early, or more than 2 prescription refills at least 3 days early. The "missed visit" behavior included either failure to present for a scheduled appointment or a same-day cancellation. We assigned patients to 1 of 2 mutually exclusive groups: any PDB (at least 1 type of PDB identified) or no PDB. Approximately half of patients with any PDB exhibited $\geq 3$ types of PDB, and we assigned them to $\mathrm{a} \geq 3 \mathrm{PDB}$ group to create a subgroup of patients with the highest level of PDB.

We further assigned patients to 1 of 2 mutually exclusive groups: those for whom the clinic was still prescribing opioids after 2 years (defined as a nonzero MED for at least 1 of the last 3 months of the study period), or those for whom the clinic was no longer prescribing opioids (defined as zero MED for all 3 of the last 3 months). 
The latter group included patients with many different reasons for no longer receiving opioid prescriptions at the clinic (e.g., the provider's judgment of decreased benefit or increased risk; the patient's decision to discontinue, move, transfer to another clinic, undergo chemical dependency treatment, or obtain opioids elsewhere; or death of the patient).

A UDT was classified as inconsistent if (1) a nonprescribed controlled substance, an illicit substance, or a metabolite of either was found in any quantity; (2) an opioid prescribed for daily use during the previous month was absent, which could reflect diversion or overuse with a subsequent period of abstinence; or (3) an opioid prescribed for daily use during the previous month was found but an expected metabolite was absent, which could occur if the prescribed opioid was consumed shortly before the test after a prolonged period of abstinence, interpreted as potentially deceptive behavior in advance of an expected UDT. The presence of tetrahydrocannabinol was interpreted as inconsistent only if chart notes indicated that marijuana use was unacceptable to the patient's provider.

\section{Analysis}

We calculated simple frequencies of the demographic and clinical characteristics of patients across the different PDB groups. Demographic characteristics, clinical characteristics, and opioidrelated process and outcome measures were compared between the no PDB and any PDB groups using $\chi^{2}$ tests and Fisher exact tests. Mean values for age, opioid dose, and number of visits in 2010 were compared between the no PDB and any PDB groups using Student $t$ test and one-way analysis of variance. Overall tests were also performed across the no PDB, 1 or 2 PDB, and $\geq 3$ PDB groups. For characteristics and clinical care measures with significant differences in the overall tests, additional comparisons were made between the no PDB group and the $\geq 3$ PDB subgroup. The study received exemption determinations from both the Western Institutional Review Board and the University of Washington Human Subjects Review Board, based on 45 CFR 46.101(b)(4), deidentification of currently existing protected health information.

\section{Results}

The study included 233 registry patients who had received opioid prescriptions both by the start of and during the 2-year study period (Figure 1). PDBs were identified in $84.1 \%$ of patients, and $\geq 3$ types of PDB were identified in $45.5 \%$. The any PDB group was significantly younger than the no PDB group (Table 1). Most patients were female, white, without identified employment, and publicly insured; there were no significant differences in these characteristics between groups. The average duration of opioid therapy in the clinic before the study period was 37.8 months, with no significant difference between groups. The prevalence of smoking, depression, and anxiety was significantly higher in the any PDB group, particularly in the $\geq 3$ PDB subgroup, compared with the no PDB group.

The clinic continued to prescribe opioids to $70.4 \%$ of the registry patients overall, with $78.4 \%, 68.9 \%$, and $62.3 \%$ in the no PDB group, the any $\mathrm{PDB}$ group, and the $\geq 3 \mathrm{PDB}$ subgroup, respectively; the difference between the no $\mathrm{PDB}$ group and the $\geq 3$ PDB subgroup was statistically significant (Table 2). Two patients initiated treatment for chemical dependence; both were in the $\geq 3$ PDB subgroup. Three patients died, 2 of whom were in the $\geq 3$ PDB subgroup. Average opioid dose (MED) was lower in the no PDB group (41.7) than the any PDB group (133.8; $P<$ $.001)$ or the $\geq 3$ PDB subgroup (169.0; $P<$ $.001)$.

UDT and treatment agreements were more common in the $\geq 3 \mathrm{PDB}$ subgroup and the any PDB group compared with the no PDB group (for UDT: $87.7 \%$ vs $48.6 \%[P<.001]$ and $76.5 \%$ vs 48.6\% $[P<.001]$, respectively); for treatment agreements: $89.6 \%$ vs $73.0 \%[P=.014]$ and $88.3 \%$ vs $73.0 \%[P=.014]$, respectively). Patients visited the clinic frequently; the average number of visits in 2010 was higher in the $\geq 3$ PDB subgroup and the any PDB group compared with the no PDB group $(9.2$ vs $4.2[P<.001]$ and 8.1 vs $4.2[P<$ $.001]$, respectively).

The most common types of PDB were inconsistent UDT, missed appointments, and early refills (Figure 2). Each of these 3 behaviors was exhibited by $>40 \%$ of registry patients during the 2 -year study period. 
Figure 1. Sample selection from a registry of patients prescribed chronic opioid therapy between December 1, 2009, and November 30, 2011. ICD-9, International Classification of Diseases, 9th Revision; PDB, problem drugrelated behavior.

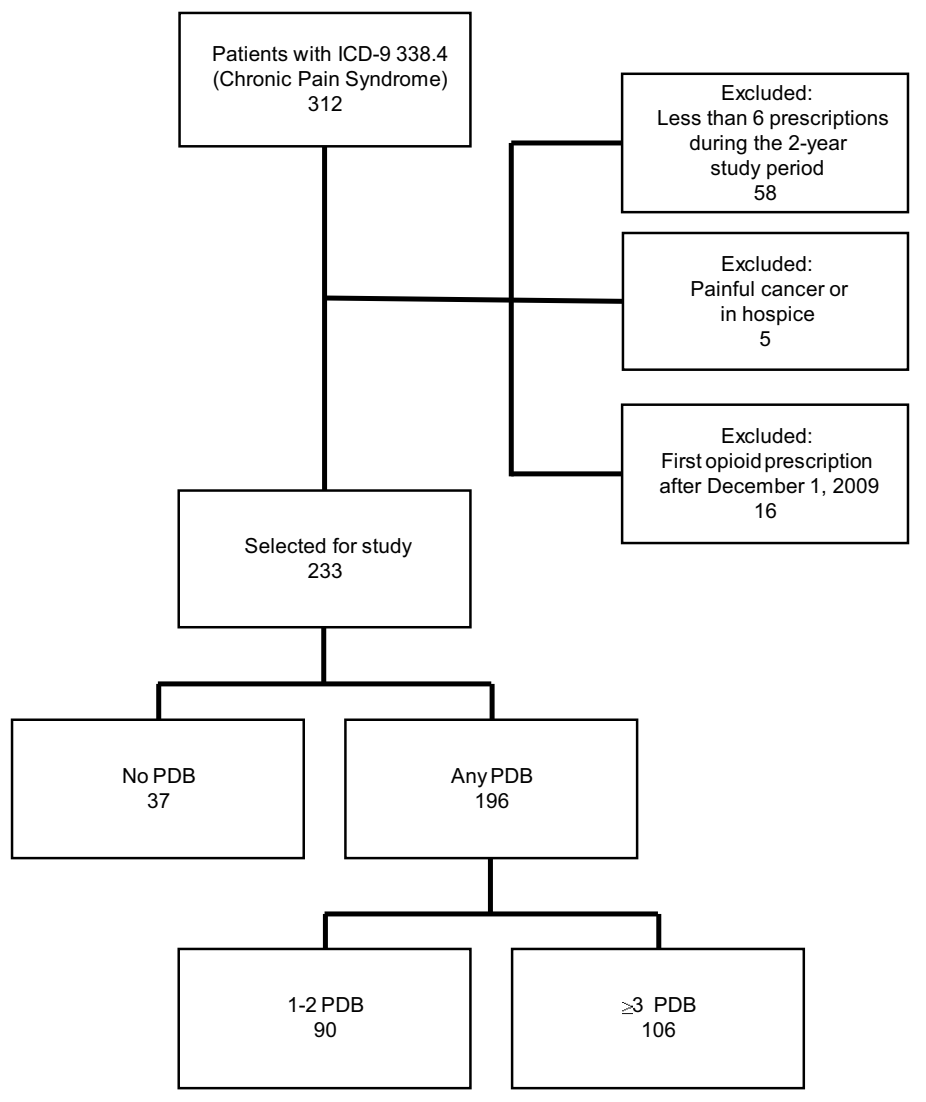

Table 1. Demographics and Clinical Characteristics by Presence of Problem Drug-Related Behavior

\begin{tabular}{|c|c|c|c|c|c|c|}
\hline & \multirow[b]{2}{*}{ No PDB } & \multirow[b]{2}{*}{ Any PDB } & \multirow[b]{2}{*}{$\geq 3 \mathrm{PDB}$} & \multirow[b]{2}{*}{ Total } & \multicolumn{2}{|c|}{$P$ Value } \\
\hline & & & & & $\begin{array}{l}\text { Any PDB vs } \\
\text { No PDB }\end{array}$ & $\begin{array}{l}\geq 3 \text { PDB vs } \\
\text { No PDB }\end{array}$ \\
\hline Patients & $37(15.9)$ & $196(84.1)$ & $106(45.5)$ & $233(100.0)$ & & \\
\hline $\begin{array}{l}\text { Age as of December 1, } 2009 \text { (years), } \\
\text { mean (SD) }\end{array}$ & $56.4(15.7)$ & $47.1(12.3)$ & $45.1(11.6)$ & $48.6(13.3)$ & $<.001$ & $<.001$ \\
\hline Female sex & $24(64.9)$ & $147(75.0)$ & $81(76.4)$ & $171(73.4)$ & .201 & - \\
\hline White & $36(97.3)$ & $183(93.4)$ & $101(95.3)$ & $219(94.0)$ & $.698^{*}$ & - \\
\hline Not employed (or unknown) & $24(64.9)$ & $140(71.4)$ & $79(74.5)$ & $164(70.4)$ & .533 & - \\
\hline Public insurance (Medicare/Medicaid) & $18(48.6)$ & $115(58.7)$ & $62(58.5)$ & $133(57.1)$ & .258 & - \\
\hline $\begin{array}{l}\text { Duration of opioid prescription (months) } \\
\text { as of December 1, 2009, mean (SD) }\end{array}$ & $38.4(15.4)$ & $37.7(15.6)$ & $38.0(16.4)$ & $37.8(15.5)$ & .793 & - \\
\hline Smoker & $11(29.7)$ & $100(51.0)$ & $62(58.5)$ & $111(47.6)$ & .017 & .003 \\
\hline Depression & $18(48.6)$ & $143(73.0)$ & $84(79.2)$ & $161(69.1)$ & .003 & $<.001$ \\
\hline Anxiety & $9(24.3)$ & 105 (53.6) & $64(60.4)$ & $114(48.9)$ & .001 & $<.001$ \\
\hline PTSD & $2(5.4)$ & $24(12.2)$ & $16(15.1)$ & $26(11.2)$ & $.390^{*}$ & - \\
\hline
\end{tabular}

Data are n (\%) unless otherwise indicated. Statistically significant $P$ values are shown in boldface.

*Calculated using the Fisher exact test.

PDB, problem drug-related behavior; PTSD, post-traumatic stress disorder; SD, standard deviation. 
Table 2. Opioid-Related Clinical Care by Presence of Problem Drug-Related Behavior

\begin{tabular}{|c|c|c|c|c|c|c|}
\hline & \multirow[b]{2}{*}{ No PDB } & \multirow[b]{2}{*}{ Any PDB } & \multirow[b]{2}{*}{$\geq 3 \mathrm{PDB}$} & \multirow[b]{2}{*}{ Total } & \multicolumn{2}{|c|}{$P$ Value } \\
\hline & & & & & $\begin{array}{l}\text { Any PDB vs } \\
\text { No PDB }\end{array}$ & $\begin{array}{l}\geq 3 \text { PDB vs } \\
\text { No PDB }\end{array}$ \\
\hline Patients & $37(15.9)$ & $196(84.1)$ & $106(45.5)$ & $233(100.0)$ & & \\
\hline Disposition & & & & & .051 & .016 \\
\hline Clinic still prescribing opioids & $29(78.4)$ & $135(68.9)$ & $66(62.3)$ & $164(70.4)$ & & \\
\hline Clinic no longer prescribing opioids* & $8(21.6)$ & $61(31.1)$ & $40(37.7)$ & $69(29.6)$ & & \\
\hline MED, mean (SD) & $41.7(55.4)$ & $133.8(273.2)$ & $169.0(319.7)$ & $119.2(253.7)$ & $<.001$ & $<.001$ \\
\hline Treatment agreement & $27(73.0)$ & $173(88.3)$ & $95(89.6)$ & $200(85.8)$ & .014 & .014 \\
\hline Had UDT & $18(48.6)$ & $150(76.5)$ & $93(87.7)$ & $168(72.1)$ & $<.001$ & $<.001$ \\
\hline Visits in 2010, mean (SD) & $4.2(3.0)$ & $8.1(6.2)$ & $9.2(6.6)$ & $7.5(6.0)$ & $<.001$ & $<.001$ \\
\hline
\end{tabular}

Data are n (\%) unless otherwise indicated. Statistically significant $P$ values are shown in boldface.

*Includes 2 patients who entered chemical dependency treatment and 3 who died.

MED, morphine-equivalent dose; PDB, problem drug-related behavior; SD, standard deviation; UDT, urine drug testing.

\section{Discussion}

PDB was pervasive among patients prescribed chronic opioid therapy at this safety net clinic. The $84.1 \%$ prevalence of $\mathrm{PDB}$ was high relative to the prevalence of problem behaviors and addiction found in other studies ( $0 \%$ to $81 \%) .{ }^{12-14}$ One important reason may be this clinic's patient population: the high proportion receiving public assistance and without employment could be associated with a higher-than-average risk of an opioid use disorder. ${ }^{15}$ Opioid use disorder is common among medical users of opioids, ${ }^{11}$ and many patients dis- playing problematic behavior may meet criteria for it. Another important reason was the study's methodology. The use of objective criteria (quantitative UDT results and chart notes) may have uncovered more PDB than the subjective criteria (e.g., interviews with providers or patients) often used in other studies. ${ }^{12-14}$ In addition, the combination of UDT results and chart notes used in this analysis may have produced a higher PDB detection rate than either one alone. ${ }^{16}$ Manual review of chart notes, sensitive criteria (e.g., a single missed visit or dose violation, absence of the prescribed drug from

Figure 2. Rates of different types of problem drug-related behavior (PDB) between December 1, 2009, and November 30, 2011, among registry patients. *Prevalence of inconsistent urine drug test (UDT) is calculated as the fraction of patients who had at least 1 UDT. ED, emergency department.

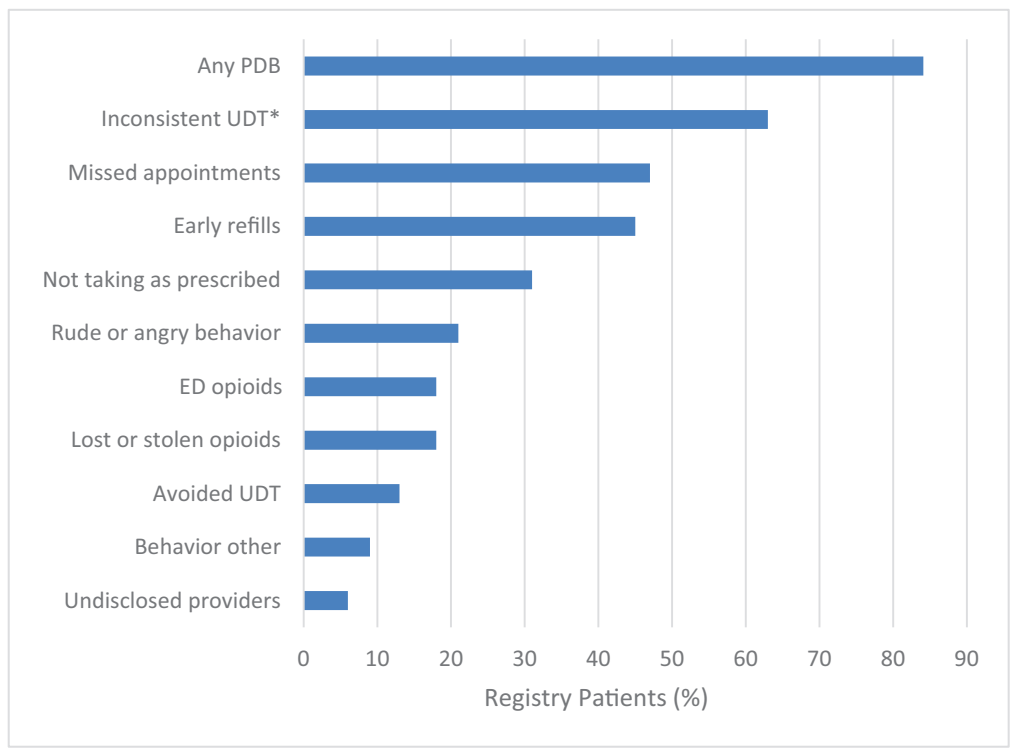


the urine), and a long study period allowed us to identify problematic behaviors that may have escaped the notice of the patients' own providers.

Demographic and clinical characteristics differed between the groups with and without PDB. Higher rates of smoking and mental illness and higher health care utilization (reflected by more frequent clinic visits) were found among patients with PDB, particularly in the subgroup with the most PDB. These characteristics would be expected among patients who are more severely affected along the broad spectrum of addictive behaviors described by Ballantyne and Stannard ${ }^{17}$ in patients prescribed opioids for chronic pain.

Patients with multiple types of PDB were less likely than those without PDB to continue receiving opioid prescriptions from the clinic at the end of the study period, despite their similar duration of opioid therapy in the clinic before the study period. The clinic's introduction of a population management approach to opioid prescribing may have increased provider awareness and recognition of PDB. For example, because the clinic began to conduct UDTs more frequently, the providers became more likely to identify inconsistent UDTs. In contrast to only $8 \%$ of patients undergoing UDT in a study of 8 primary care clinics over 4 years, ${ }^{18}$ this clinic achieved an overall $72.1 \%$ rate of UDT over 2 years. After a similar multifaceted opioid risk reduction initiative, an integrated health system increased its rate of UDT from $7 \%$ to $50 \%$ after 1 year. ${ }^{19}$ Another possible reason for reduced prescribing to patients with more PDB is that more of these patients chose to obtain opioids elsewhere after close monitoring was begun.

At the end of the 2-year study period, the majority of registry patients, including the majority of those with multiple types of PDB, were still being prescribed opioids. Chronic prescription of opioids to patients with PDB is widespread in primary care. ${ }^{20}$ Patients and providers both consider pain to be the primary issue for these patients. ${ }^{7}$ Providers may continue prescribing opioids for pain even when they recognize PDB and acknowledge a comorbid opioid use disorder.

Patients who are no longer prescribed opioids may seek prescription opioids elsewhere. They may find another clinic, obtain prescription opioids illicitly, or turn to heroin. ${ }^{11,21}$ For patients with pain and a recognized comorbid opioid use disorder, providers are therefore in the predicament of risk- ing harm by either continuing or discontinuing prescriptions. Continuing prescriptions reinforces maladaptive behaviors and risks the adverse consequences of uncontrolled opioid use. Discontinuing prescriptions risks poorly controlled pain, a loss of continuity of care and the adverse consequences of illicit drug use. In either case, the risks include death from overdose.

Providers have had little guidance on how to help these patients beyond referring them elsewhere for chemical dependency treatment when PDB is extreme. The Philadelphia Veterans Administration created an effective population management program within the clinic for patients with problematic behaviors; they offered provider and patient education, a dedicated team of specialists (a nurse practitioner and a clinical pharmacist), more frequent patient visits, and biweekly review of cases by a multidisciplinary pain team including an addiction psychiatrist and physiatrist. ${ }^{22}$ A complementary approach is endorsed by the Centers for Disease Control and Prevention: "clinicians should offer or arrange evidence-based treatment (usually medication-assisted treatment with buprenorphine or methadone in combination with behavioral therapies) for patients with opioid use disorder." ${ }^{\circ}$ There is ample evidence that medication-assisted treatment is the most effective way to retain these patients in treatment and improve social function. ${ }^{23}$ Recent evidence suggests it reduces the risk of overdose death, ${ }^{24}$ and buprenorphine may reduce pain severity. ${ }^{25,26}$ Unfortunately, referral is an inadequate plan for many. Patients often shun methadone clinics because of stigma ${ }^{27}$ and inconvenience (particularly outside urban areas), ${ }^{28}$ and the need for buprenorphine prescribers far outstrips the supply. ${ }^{29}$

This report adds a new dimension to the sparse literature on primary care clinics using populationbased opioid safety initiatives. ${ }^{19,22,30,31}$ Clinics implementing safety initiatives should consider ensuring access to evidence-based treatment for patients with an opioid use disorder in order to protect those patients from foreseeable harms when opioid prescriptions are discontinued. ${ }^{11}$ To this end, they can encourage and arrange for their physicians to become trained and certified to prescribe buprenorphine to their patients with an opioid use disorder. $^{32}$

This study has several limitations. The PDB designations reflect the interpretation of a single 
reviewer. The generalizability of the study is limited by its conduct in a single clinic. The clinic's quality improvement project may have altered the prevalence of $\mathrm{PDB}$, though we could not evaluate this with our data. The failure of some patients to undergo UDT suggests our PDB prevalence estimate may be low. Additional risk mitigation strategies such as pill counting or the use of the state Prescription Monitoring Program (which was not available at the time of the project) might have further increased detection of PDB. Some patients with multiple opioid prescriptions during the study period were not tracked in the registry since they were not considered by their providers to be chronic opioid users; these individuals may have differed from registry patients in important ways. Finally, the study was not able to examine the longer-term outcome of patients whose opioids were no longer prescribed by the clinic; this is an important topic for further research.

\section{Conclusion}

This study identified a high rate of PDB among patients receiving chronic opioid therapy in a safety net primary care practice. Patients with the greatest likelihood of an opioid use disorder were least likely to continue receiving opioid prescriptions from the clinic. With increasing awareness of the rising risks of opioid addiction and overdose death, medical clinics and their providers may be seeking methods to reduce opioid prescribing. Population management strategies and guideline-based monitoring may be helpful for clinics to identify patients who have an opioid use disorder. To reduce the morbidity and mortality of this chronic disease, however, reduced opioid prescribing must be accompanied by increased access to evidence-based treatment for patients at risk.

The authors gratefully acknowledge support from the Washington State Life Science Discovery Fund (WSU-LSDF subcontract 109212-G002562; Principal Investigator, John Roll) and the University of Washington School of Medicine Medical Student Research Training Program and the Department of Family Medicine. The authors thank the clinic's "Pain Gang" for developing and implementing the clinic protocol: Rebecca BodleShingu, ARNP; Cynthia Chenot-Potter, RN; Leslie Edwards; Elizabeth McDonnell, RN; Jessica Waunch; and Kristin White.

\section{References}

1. Centers for Disease Control and Prevention (CDC). Vital signs: overdoses of prescription opioid pain re-
lievers-United States, 1999-2008. MMWR Morb Mortal Wkly Rep 2011;60:1487-92.

2. Chou R, Fanciullo GJ, Fine PG, et al; American Pain Society-American Academy of Pain Medicine Opioids Guidelines Panel. Clinical guidelines for the use of chronic opioid therapy in chronic noncancer pain. J Pain 2009;10:113-30.

3. Federation of State Medical Boards. Model policy on the use of opioid analgesics in the treatment of chronic pain. Available from: http://www.fsmb.org/ Media/Default/PDF/FSMB/Advocacy/pain_policy_ july2013.pdf. Accessed June 26, 2016.

4. Washington State Agency Medical Directors Group (AMDG). Interagency guideline on prescribing opioids for pain. Available from: http://www. agencymeddirectors.wa.gov/Files/2015AMDGO pioidGuideline.pdf. Accessed June 26, 2016.

5. Johnson H, Paulozzi L, Porucznik C, Mack K, Herter B; Hal Johnson Consulting and Division of Disease Control and Health Promotion, Florida Department of Health. Decline in drug overdose deaths after state policy changes - Florida, 2010-2012. MMWR Morb Mortal Wkly Rep 2014;63:569-74.

6. Dowell D, Haegerich TM, Chou R. CDC guideline for prescribing opioids for chronic pain - United States, 2016. MMWR Recomm Rep 2016;65:1-49.

7. Ballantyne JC, Sullivan MD, Kolodny A. Opioid dependence vs. addiction: a distinction without a difference? Arch Intern Med 2012;172:1342-3.

8. Højsted J, Nielsen PR, Guldstrand SK, Frich L, Sjøgren P. Classification and identification of opioid addiction in chronic pain patients. Eur J Pain 2010; 14:1014-20.

9. Yanni LM, Weaver MF, Johnson BA, Morgan LA, Harrington SE, Ketchum JM. Management of chronic nonmalignant pain: a needs assessment in an internal medicine resident continuity clinic. J Opioid Manag 2008;4:201-11.

10. Corrigan C, Desnick L, Marshall S, Bentoy N, Rosenblatt RA. What can we learn from first-year medical students' perceptions of pain in the primary care setting? Pain Med 2011;12:1216-22.

11. Kolodny A, Courtwright DT, Hwang CS, et al. The prescription opioid and heroin crisis: a public health approach to an epidemic of addiction. Annu Rev Public Health 2015;36:559-74.

12. Ballantyne JC, LaForge KS. Opioid dependence and addiction during opioid treatment of chronic pain. Pain 2007;129:235-55.

13. Højsted J, Sjøgren P. Addiction to opioids in chronic pain patients: a literature review. Eur J Pain 2007; 11:490-518.

14. Vowles KE, McEntee MK, Julnes PS, Frohe T, Ney JP, van der Goes DN. Rates of opioid misuse, abuse, and addiction in chronic pain: a systematic review and data synthesis. Pain 2015;156:569-76.

15. Becker WC, Fiellin DA, Merrill JO, et al. Opioid use disorder in the United States: insurance status and 
treatment access. Drug Alcohol Depend 2008;94: 207-13.

16. Katz NP, Sherburne S, Beach M, et al. Behavioral monitoring and urine toxicology testing in patients receiving long-term opioid therapy. Anesth Analg 2003;97:1097-102.

17. Ballantyne JC, Stannard C. New addiction criteria: diagnostic challenges persist in treating patients with opioids. Pain Clin Updates 2013;21:1-7.

18. Starrels JL, Becker WC, Weiner MG, Li X, Heo M, Turner BJ. Low use of opioid risk reduction strategies in primary care even for high risk patients with chronic pain. J Gen Intern Med 2011;26:958-64.

19. Turner JA, Saunders K, Shortreed SM, et al. Chronic opioid therapy risk reduction initiative: impact on urine drug testing rates and results. J Gen Intern Med 2014;29:305-11.

20. Cheatle MD, Klocek JW, McLellan AT. Managing pain in high-risk patients within a patient-centered medical home. Transl Behav Med 2012;2:47-56.

21. Rudd RA, Paulozzi LJ, Bauer MJ, et al; Centers for Disease Control and Prevention (CDC). Increases in heroin overdose deaths - 28 states, 2010 to 2012. MMWR Morb Mortal Wkly Rep 2014;63:849-54.

22. Wiedemer NL, Harden PS, Arndt IO, Gallagher RM. The opioid renewal clinic: a primary care, managed approach to opioid therapy in chronic pain patients at risk for substance abuse. Pain Med 2007; 8:573-84.

23. Bart G. Maintenance medication for opiate addiction: the foundation of recovery. J Addict Dis 2012; 31:207-25.

24. Pierce M, Bird SM, Hickman M, et al. Impact of treatment for opioid dependence on fatal drug-related poisoning: a national cohort study in England. Addiction 2015;111:298-308.

25. Daitch D, Daitch J, Novinson D, Frey M, Mitnick C, Perfolizzi J Jr. Conversion from high-dose full- opioid agonists to sublingual buprenorphine reduces pain scores and improves quality of life for chronic pain patients. Pain Med 2014;15:2087-94.

26. Roux P, Sullivan MA, Cohen J, et al. Buprenorphine/naloxone as a promising therapeutic option for opioid abusing patients with chronic pain: reduction of pain, opioid withdrawal symptoms, and abuse liability of oral oxycodone. Pain 2013;154:1442-8.

27. Earnshaw V, Smith L, Copenhaver M. Drug addiction stigma in the context of methadone maintenance therapy: an investigation into understudied sources of stigma. Int J Ment Health Addict 2013; 11:110-22.

28. Substance Abuse and Mental Health Services Administration. 2011 Opioid Treatment Program Survey: data on substance abuse treatment facilities with OTPs. BHSIS Series S-65, HHS publication no. (SMA) 14-4807. Rockville, MD: Substance Abuse and Mental Health Services Administration, 2013. Available from: http://www.samhsa.gov/data/sites/ default/files/OTP2011_Web/OTP2011_Web/OTP 2011_Web.pdf. Accessed June 26, 2016.

29. Rosenblatt RA, Andrilla CH, Catlin M, Larson EH. Geographic and specialty distribution of US physicians trained to treat opioid use disorder. Ann Fam Med 2015;13:23-6.

30. Westanmo A, Marshall P, Jones E, Burns K, Krebs EE. Opioid dose reduction in a VA health care system-implementation of a primary care populationlevel initiative. Pain Med 2015;16:1019-26.

31. Weimer MB, Hartung DM, Ahmed S, Nicolaidis C. A chronic opioid therapy dose reduction policy in primary care. Subst Abus 2016;37:141-7.

32. Drug Addiction Treatment Act of 2000. Publ L No. 106-310, Stat 1101. Available from: http:// www.samhsa.gov/medication-assisted-treatment/ legislation-regulations-guidelines\#DATA-2000. Accessed June 26, 2016. 